\title{
On the Averaging of a Class of Hybrid Systems
}

\author{
Luigi Iannelli \\ Dept. of Computer and Systems \\ Engineering \\ University of Napoli Federico II \\ Napoli, Italy \\ luiannel@unina.it
}

\author{
Karl Henrik Johansson \\ Signals, Sensors \& Systems \\ Royal Institute of Technology \\ Stockholm, Sweden \\ kallejes3.kth.se
}

\author{
Ulf T. Jönsson \\ Optimization and Systems \\ Theory \\ Royal Institute of Technology \\ Stockholm, Sweden \\ ulfj@math.kth.se
}

\author{
Francesco Vasca \\ Dept. of Engineering \\ University of Sannio \\ Benevento, Italy \\ vasca@unisannio.it
}

\begin{abstract}
Modeling abstraction and time-scale separation in the design of complex systems often leads to hybrid dynamics. Discontinuities in the continuous evolution of a hybrid system may however create difficulties in the formal analysis, as well as in numerical simulation and verification. Here we study a class of hybrid systems that are excited by high-frequency external signals. These systems arise in the modeling of switched power converters, mechanical systems with friction and quantized systems. For a quite general class of excitation signals, an averaging result is shown stating that the hybrid system can be approximated by a Lipschitzcontinuous system. The approximation is in the order of the maximal repetition interval of the excitation signal.
\end{abstract}

\section{INTRODUCTION}

To find abstract models of technological phenomena is essential in many areas of engineering. For control system design, a good model is one that is complex enough to capture the important system characteristics (dynamics, disturbances, measurement noise etc.), but simple enough to allow application of existing analysis and design methods. Hybrid systems provide a tempting framework, which extracts desired properties of a system while ignoring irrelevant details. Due to discontinuities in the continuous-time evolution of hybrid systems, the formal analysis of these systems is sometimes tricky. Over the past decade, there has been a major development in the understanding of the dynamic behavior of hybrid and switched systems, such as existence and uniqueness of discrete and continuous evolution (e.g., [1], [2]) and Zeno phenomena (e.g., [3]). Still the dynamics of these systems are not fully understood and several interesting phenomena have recently been studied for very simple hybrid systems [4], [5].

In this paper we study a class of hybrid systems with high-frequency external excitation. The study is motivated by applications in mechanical friction [6], such as stickslip drives [7], power electronics [8], such as pulse-width modulated converters [9], feedback systems [10] and quantized systems [11]. For instance, excitation signals are used for power electronics converters in order to ensure a finite and constant switching frequency and in relay feedback systems for quenching undesired oscillations such as limit cycles. Following our earlier work on dithered feedback

The work by L. Iannelli and F. Vasca was supported by EC within the SICONOS project (IST2001-37172). The work by K. H. Johansson and U. Jönsson was supported the Swedish Research Council and by EC within the RECSYS project. systems [12], we consider a quite general class of piecewise smooth systems in this paper. The systems are excited by so called $F$-repetitive high-frequency excitation signals, introduced by Zames and Shneydor [13] as being signals with periodic amplitude distribution functions. These signals are of rather general form (e.g., not necessarily periodic), and well tailored to the theoretical analysis. The main result of the paper is an averaging result for this class of hybrid systems. It states that the (typically discontinuous) hybrid system can be approximated by a Lipschitz-continuous system. The approximation is in the order of the maximal repetition interval of the excitation signal. Averaging is a classical mathematical tool for analyzing time-scale separation in dynamical systems. Related work on averaging applied to discontinuous and hybrid system was recently presented in [7], [10], [14], while the case of Lipschitzcontinuous systems was thoroughly treated already in the 70's [13].

The outline of the paper is as follows. The problem formulation and some preliminaries are given in Section II. The main result is then presented in Section III, where it is stated that the averaged system is Lipschitz continuous and that its solution is at most of order $p$ from any corresponding solutions of the hybrid system, where $p$ is the maximal repetition interval of the excitation signals. Section IV presents three illustrative examples. Some concluding remarks are given in Section V.

\section{PRELIMINARIES}

\section{A. Autonomous hybrid system}

Consider a convex polyhedron $\Omega \subset \mathbb{R}^{n}$, defined as the set $\Omega \triangleq\{x \mid A x+B \preceq 0\}$ with $x \in \mathbb{R}^{n}, A \in \mathbb{R}^{m \times n}$ and $B \in$ $\mathbb{R}^{n}, n, m>0$. Here the symbol “ $\preceq$ " means componentwise inequality. Let us consider a minimal cover of $\Omega$ that consists of $N \geq 1$ convex polyhedra $\omega_{1}, \ldots, \omega_{N} \subset \Omega$ with disjoint interior defined as

$$
\omega_{q} \triangleq\left\{x \mid A_{q} x+B_{q} \preceq 0\right\}, \quad q=1, \ldots, N,
$$

with $A_{q} \in \mathbb{R}^{m \times n}$ and $B_{q} \in \mathbb{R}^{n}$. Introduce indicator functions $I_{q}: \mathbb{R}^{n} \mapsto\{0,1\}, q=1, \ldots, N$, for these sets:

$$
I_{q}(x)= \begin{cases}1, & x \in \omega_{q} \\ 0, & \text { otherwise }\end{cases}
$$


A class of hybrid systems can then be defined as

$$
\dot{x}(t)=\sum_{q=1}^{N} I_{q}(x(t)) f_{q}(x(t)), \quad x(0)=x_{0},
$$

where each vector field $f_{q}$ is supposed to have Lipschitz constant $L_{f}$ in $\Omega$. The class of systems we deal with is obtained from (1) by introducing an external excitation signal. Before showing that, for our further development, it will be convenient to have a more explicit formulation of the indicator function in the system (1).

Proposition 2.1: Let $a_{q}^{j}$ be the $j$-th row of the $A_{q}$ matrix and $b_{q}^{j}$ be the $j$-th component of the $B_{q}$ vector. Further, let $n(z)$ be the reverse unit step function $(=1$ if $z \leq 0$ and 0 if $z>0$ ). Then system (1) can be written in the following way:

$$
\dot{x}(t)=\sum_{q=1}^{N} n\left[g_{q}(x(t))\right] f_{q}(x(t)), \quad x(0)=x_{0},
$$

where

$$
g_{q}(x)=\max _{i=1, \ldots, m}\left(a_{q}^{i} x+b_{q}^{i}\right) .
$$

is Lipschitz continuous with Lipschitz constant $L_{g}=$ $\max _{i}\left|a_{q}^{i}\right|$.

Proof: Omitted.

\section{B. Excitation signal}

The excitation signal $\delta:[0, \infty) \mapsto \mathbb{R}$ is an highfrequency signal, which is characterized through its amplitude distribution function (ADF):

Definition 2.1: The amplitude distribution function of $\delta$ : $[0, \infty) \mapsto \mathbb{R}$ on $\left(t_{0}, t_{1}\right)$ is the real valued function $F_{\delta}: \mathbb{R} \mapsto$ $[0,1]$

$$
F_{\delta}(\xi)=\frac{1}{t_{1}-t_{0}} \mu\left\{t \in\left(t_{0}, t_{1}\right): \delta(t) \leq \xi\right\}
$$

where $\mu$ denotes Lebesgue measure.

We introduce the notion that the amplitude distribution function of $\delta$ is $F$-repetitive, following Zames and Shneydor [13].

Definition 2.2: A signal $\delta:[0, \infty) \mapsto \mathbb{R}$ is $F$-repetitive if there exists an unbounded sequence $\left\{t_{k}\right\}, 0=t_{0}<t_{1} \ldots$, of partition points such that

1) the maximal repetition interval $p \triangleq \max _{k}\left(t_{k}-t_{k-1}\right)$ is bounded

2) $F_{\delta}$ on $\left(t_{k}, t_{k-1}\right)$ is equal to $F_{\delta}$ on $\left(t_{0}, t_{1}\right)$ for all $k>0$.

Note that an $F$-repetitive $\delta$ needs not itself be periodic. We make following standing assumption.

Assumption 2.1: The signal $\delta$ is $F$-repetitive, bounded by $M_{\delta}$ (i.e., $\|\delta\|_{\infty} \leq M_{\delta}$ ), and its amplitude distribution function $F_{\delta}$ is absolutely continuous and Lipschitz continuous with

$$
\left|F_{\delta}\left(\xi_{1}\right)-F_{\delta}\left(\xi_{2}\right)\right| \leq L_{F}\left|\xi_{1}-\xi_{2}\right| .
$$

Remark 1: We will use that the ADF is monotonically increasing. We will sometimes also use that Lipschitz continuity of $F_{\delta}$ is equivalent to the existence of a bounded density function $f_{\delta}$ such that $F_{\delta}(\xi)=\int_{-\infty}^{\xi} f_{\delta}(\zeta) \mathrm{d} \zeta$ and $\operatorname{ess}_{\sup _{\zeta}} f_{\delta}(\zeta)=L_{f}$. Note that $f_{\delta}(\zeta)$ is positive since $F_{\delta}$ is monotonically increasing.

Remark 2: Signals that fulfill the assumption on the amplitude distribution function include periodic triangular and sawtooth signals, while square wave signals have discontinuous amplitude distribution function, see [10], [12].

\section{Hybrid system with excitation}

The hybrid system under investigation is obtained from (1) by introducing an $F$-repetitive excitation signal $\delta_{q}$ for each mode $q=1, \ldots, N$ :

$$
\dot{x}(t)=\sum_{q=1}^{N} \tilde{I}_{q}\left(x(t), \delta_{q}(t)\right) f_{q}(x(t)), \quad x(0)=x_{0}
$$

where

$\tilde{I}_{q}\left(x, \delta_{q}\right)= \begin{cases}1, & x \in \tilde{\omega}_{q}(t) \triangleq\left\{x \mid A_{q} x+B_{q}+\delta_{q}(t) \mathbf{1} \preceq 0\right\}, \\ 0, & \text { otherwise. }\end{cases}$

where $\mathbf{1}=\left[\begin{array}{lll}1 & \ldots & 1\end{array}\right]^{T}$. By Proposition 2.1 this system can be rewritten as

$$
\dot{x}(t)=\sum_{q=1}^{N} n\left[g_{q}(x(t))+\delta_{q}(t)\right] f_{q}(x(t)), \quad x(0)=x_{0} .
$$

Throughout the paper we make the assumption that system (5) is well-posed in the sense that a solution according to the following definition always exists. We denote a solution $x(t)$ and sometimes $x\left(t, x_{0}\right)$.

Definition 2.3: A solution $x(t)$ of (5) is an absolutely continuous function $x:\left[0, t_{f}\right) \mapsto \Omega$ that satisfies the equation

$$
x(t)=x(0)+\sum_{q=1}^{N} \int_{0}^{t} \tilde{I}_{q}\left(x(s), \delta_{q}(s)\right) f_{q}(x(s)) \mathrm{d} s,
$$

almost everywhere. Further, we assume that $x(t)$ does not belong to any $\partial \tilde{\omega}_{j}$ for a time interval of Lebesgue measure different from zero and that the sequence of (switching) time instants $\left\{t_{i}\right\}$ such that $x\left(t_{i}\right)$ belongs to some $\partial \tilde{\omega}_{j}$ does not have an accumulation point.

Since the definition of solution does not incorporate sliding modes and accumulation points, in some cases solutions may not exist. Furthermore the value of the vector field on $\partial \tilde{\omega}_{q}$ does not affect the solution since by assumption $x(t) \in$ $\partial \tilde{\omega}_{q}$ only at distinct points in time. A possible interpretation of the influence of the excitation signals on the behavior of the hybrid system can be obtained by considering the effects of $\delta_{q}$ on the boundaries of the polyhedron $\omega_{q}$ with $q=1, \cdots, N$. One can imagine that each $\delta_{q}$ induces a timevarying, or vibrating, polyhedron $\tilde{\omega}_{q}$ whose boundaries are not static.

Note that the union of the polyhedra $\tilde{\omega}_{1}, \cdots, \tilde{\omega}_{N} \in \Omega$ will not necessarily be a cover of $\Omega$ : it could exist some time 
interval during which $x \notin \tilde{\omega}_{q}$ for all $q$, i.e., $\tilde{I}_{q}\left(x, \delta_{q}\right)=0$ for all $q$. There could also exist time intervals during which for some $i \neq j$ the interiors of $\tilde{\omega}_{i}$ and $\tilde{\omega}_{j}$ are not disjoint. In all these situations, the solution is typically still well defined. However, such situations are typically avoided in real applications. For instance, in pulse-width modulated power converters, both the excitation signals $\delta_{q}$ and the functions $g_{q}(x)$ are chosen so that there is only one active mode almost everywhere. From a more general point of view, there are cases when well-posedness depends critically on the choice the excitation signals. In Section IV we illustrate this dependency with a couple of simple examples.

\section{Averaged system}

Define the averaged indicator function $\mathcal{A}_{q}(x): \mathbb{R}^{n} \mapsto$ $[0,1]$ as

$$
\mathcal{A}_{q}(x) \triangleq \int_{\mathbb{R}} \tilde{I}_{q}(x, \xi) \mathrm{d} F_{\delta_{q}}(\xi),
$$

where the integral is a Lebesgue-Stieltjes integral. By using our assumptions and Proposition 2.1 we get

$$
\mathcal{A}_{q}(x)=\mathcal{N}_{q}\left(g_{q}(x)\right)=\int_{\mathbb{R}} n\left(g_{q}(x)+\xi\right) \mathrm{d} F_{\delta_{q}}(\xi) .
$$

Averaging (5) gives

$$
\begin{aligned}
\dot{w}(t) & =\sum_{q=1}^{N} \mathcal{A}_{q}(w(t)) f_{q}(w(t)) \\
& =\sum_{q=1}^{N} \mathcal{N}_{q}\left[g_{q}(w(t))\right] f_{q}(w(t)), \quad w(0)=w_{0},
\end{aligned}
$$

In Section III it is shown that the averaged system approximates the behavior of system (6) for a sufficiently small maximal repetition interval for the excitation signal $\delta$. The importance of this result is that the averaged system can be shown to be Lipschitz continuous and is thus well suited for mathematical analysis and simulation.

\section{Averaging Theorem}

The main results are presented in this section.

Theorem 3.1: The averaged nonlinearity in (9) is Lipschitz-continuous.

Proof: In the following we will neglect the dependence on the mode $q$. The averaged unit step function $\mathcal{N}(z)$ is computed as

$$
\mathcal{N}(z)=\int_{\mathbb{R}} n(z+\xi) \mathrm{d} F_{\delta}(\xi)=\int_{\xi \leq-z} d F_{\delta}(\xi)=F_{\delta}(-z) .
$$

Thus $\mathcal{N}(z)$ is Lipschitz continuous in $z$ with Lipschitz constant

$$
L_{F}:=\operatorname{ess} \sup _{\zeta} f_{\delta_{q}}(\zeta)
$$

Since $g$ is Lipschitz from Proposition 2.1,

$$
\left|\mathcal{N}\left(g\left(x_{1}\right)\right)-\mathcal{N}\left(g\left(x_{2}\right)\right)\right| \leq L_{F} L_{g}\left|x_{1}-x_{2}\right| .
$$

Furthermore we can derive the boundedness of $\mathcal{N}$ since

$$
|\mathcal{N}(z)|=F_{\delta}(-z) \leq 1 .
$$

The result implies that the averaged system in (10) has a Lipschitz continuous right hand side and thus there exists a unique Lipschitz-continuous solution of the averaged system on any finite time horizon (as long as we stay inside the domain of definition $\Omega$ ). We now have the following approximation result.

Theorem 3.2: Consider the hybrid system (5) and the averaged system (10). For any given $T>0$ and $x_{0} \in \Omega$,

$$
x\left(t, x_{0}\right)-w\left(t, x_{0}\right)=O(p), \quad \forall t \in[0, T],
$$

where $p$ is the maximal repetition interval of $F_{\delta}$.

Proof: This was proven for the case of periodic excitation signals in [12]. All details for the generalization to $F$-repetitive signals is given in [15].

\section{ILLUSTRATIVE EXAMPLES}

\section{A. Relay feedback systems}

Let us consider the relay feedback system

$$
\dot{x}=L x+\gamma \operatorname{sgn}\left(h^{T} x+\delta\right)
$$

with

$$
L=\left[\begin{array}{cc}
-2 & -1 \\
1 & 0
\end{array}\right], \quad \gamma=\left[\begin{array}{l}
1 \\
0
\end{array}\right], \quad h^{T}=\left[\begin{array}{ll}
1 & -1
\end{array}\right] .
$$

The system (13) without external excitation signal, i.e $\delta(t) \equiv 0$, can be modelled as a hybrid system on the form (1) with $\Omega=\omega_{1} \cup \omega_{2}$, where $\omega_{1}=\left\{x \in \mathbb{R}^{n}:-h^{T} x \leq\right.$ $0\}, \omega_{2}=\left\{x \in \mathbb{R}^{n}: h^{T} x \leq 0\right\}$,

$$
f_{1}(x)=L x+\gamma \quad \text { and } \quad f_{2}(x)=L x-\gamma
$$

The system (13) with external excitation signal, can be modelled on the form (5) in which the same excitation signal is used for both polyhedra.

When no external excitation is present $(\delta(t) \equiv 0)$, the relay feedback system presents a limit cycle as reported in Figure 1(a). The signal $-h^{T} x$ is plotted for a solution with initial condition $x_{0}=\left[\begin{array}{ll}2 & 1\end{array}\right]^{T}$. If we apply a triangle excitation signal $\delta$ with amplitude $M_{\delta}=1$ and period $p=1$, the limit cycle in Figure 1(a) is reduced as shown in Fig. 1(b). Hence, the external signal in a sense attenuates the oscillations present in the original system. Fig. 1(b) shows also the output $-h^{T} w$ of the averaged system. If we decrease the period $p$ (e.g. $p=1 / 50$ ) the averaged system and the relay feedback system have practically identical outputs. Hence the averaged system provides an accurate approximation of the relay feedback system for $p=1 / 50$. Fig. 1(b) shows the responses when the excitation signal has a larger period: $p=1$. The responses are no longer close and the output of the relay feedback system (solid) is oscillating. This confirms that the period $p$ is related to how accurately the averaged system approximates the hybrid system with the excitation signal. 


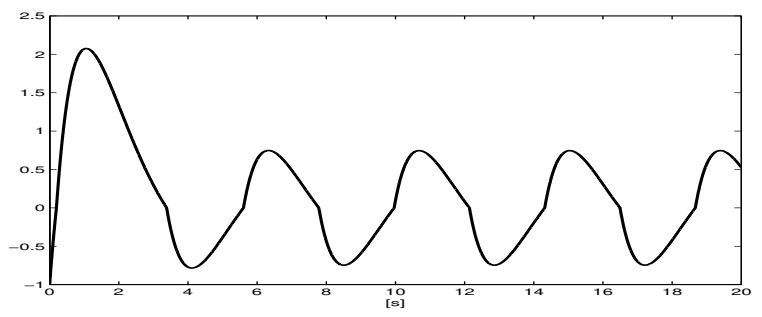

(a) Output $-h^{T} x$ of the relay feedback system (13) with (14) but without excitation signal $(\delta \equiv 0)$.

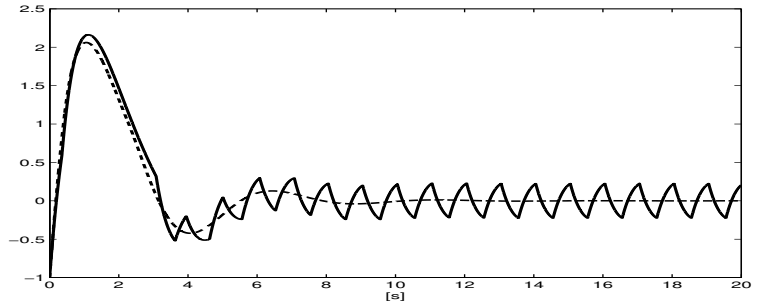

(b) Outputs of the relay feedback system (13) (solid) and the averaged system (dashed). Note the deviation between the responses. If we decrease the period (e.g. $p=1 / 50$ ) the responses are almost identical.

Fig. 1. Outputs of the relay feedback system without and with the excitation signal, and the averaged system.

\section{B. Well-Posedness?}

We now extend the previous example in order to make some considerations on the well-posedness of the hybrid system (5). We first consider two different $p$-periodic excitation signals with amplitude $M_{\delta}$

$$
\delta_{1}(t)= \begin{cases}\frac{4 M_{\delta}}{p} t, & t \in[0, p / 4] \\ -\frac{4 M_{\delta}}{p} t+2 M_{\delta}, & t \in[p / 4,3 p / 4] \\ \frac{4 M_{\delta}}{p} t-4 M_{\delta}, & t \in[3 p / 4, p]\end{cases}
$$

and $\delta_{2}(t)=\delta_{1}(t-p / 4)$. Then points near the hyperplane $H=\left\{x: h^{T} x=0\right\}$ can belong to either of the two modes, both modes, or no mode. By introducing the vibrating polyhedra

$$
\begin{aligned}
& \tilde{\omega}_{1}(t)=\left\{x \in \mathbb{R}^{n}:-h^{T} x+\delta_{1}(t) \leq 0\right\} \\
& \tilde{\omega}_{2}(t)=\left\{x \in \mathbb{R}^{n}: h^{T} x+\delta_{2}(t) \leq 0\right\}
\end{aligned}
$$

we obtain the excited dynamics

$$
\begin{aligned}
\dot{x}(t)= & (L x(t)+\gamma) \tilde{I}_{1}\left(x(t), \delta_{1}(t)\right) \\
& +(L x(t)-\gamma) \tilde{I}_{2}\left(x(t), \delta_{2}(t)\right) .
\end{aligned}
$$

We see that for example

$$
x=0 \in \begin{cases}\tilde{\omega}_{2}(t), & t \in[0, p / 4] \\ \tilde{\omega}_{1}(t), & t \in[p / 2,3 p / 4] \\ \tilde{\omega}_{1}(t) \cap \tilde{\omega}_{2}(t), & t \in[3 p / 4, p]\end{cases}
$$

and in particular $x=0$ does not belong to either of $\tilde{\omega}_{1}$ and $\tilde{\omega}_{2}$ for $t \in[p / 4, p / 2]$. If the frequency of the excitation signals is high we can expect that solutions within a distance
$M_{\delta}$ from the hyperplane $H$ will have a high frequency oscillation due to the rapid switching between the polyhedra. This example shows that even a simple hybrid system with an external excitation may have very complicated behaviour. However, under the assumption that (15) is well-posed it can be approximated by the averaged dynamics. In particular, it is easy to see that in this case (10) can be written as the saturated system

$$
\dot{w}(t)=L x+\gamma \operatorname{sat}\left(\frac{h^{T} w}{M_{\delta}}\right) .
$$

The question is when the excited system (15) will be well-posed. The dynamics is affine in the interior of the vibrating polyhedra $\tilde{\omega}_{1}(t)$ and $\tilde{\omega}_{2}(t)$ so the solution can be continued until we reach either of the boundaries $\partial \tilde{\omega}_{1}$ or $\partial \tilde{\omega}_{2}$. Suppose $x(t) \in \operatorname{int} \tilde{\omega}_{1}(t) \cap \Omega$, where $\Omega$ is a bounded open set. Then the state vector has a Lipschitz constant $L_{x} \leq \sup _{x \in \Omega}|L x \pm \gamma|$. We will show that if the period $p$ is sufficiently small then $x(t)$ cannot belong to $\partial \tilde{\omega}_{1}$ for a time interval of Lebesgue measure different from zero. Thus, if the state reaches this switching boundary it will immediately be moved inside a polyhedron region thus allowing the continuation of the solution. This shows that the solution can be continued until it reaches the boundary of $\Omega$.

To illustrate that our arguments hold for more general situations we assume that the excitation signals are general $F$-repetitive signals satisfying Assumption 2.1 with the addition that they are piecewise continuous. Let us consider the particular realization

$$
\delta_{1}(t)=F_{\delta_{1}}^{-1}\left(t /\left(t_{k}-t_{k-1}\right)\right), \quad t \in\left(t_{k-1}, t_{k}\right),
$$

This signal is strictly monotonically increasing in each repetition interval and it thus has the smallest possible slope. We will use that the slope of this excitation signal has a lower bound different from zero and this construction is thus the worst case excitation.

To derive the minimum slope we consider the derivative

$$
\frac{d}{d t} \delta_{q}(t)=\frac{1}{\left(t_{k}-t_{k-1}\right) F_{\delta_{1}}^{\prime}\left(\delta_{1}(t)\right)}
$$

which is well defined almost everywhere. Hence

$$
\min _{t}\left|\frac{d}{d t} \delta_{1}(t)\right| \geq \frac{1}{p \cdot \operatorname{ess} \sup _{\zeta} f_{\delta_{1}}(\zeta)}=: L_{\delta}>0
$$

We will assume that

$$
p<1 /\left(|h| L_{x} \cdot \operatorname{ess} \sup _{\zeta} f_{\delta}(\zeta)\right)
$$

Assume $x(t) \in \partial \tilde{\omega}_{1}(t)$ on the interval $I=\left(t_{1}-\epsilon, t_{2}+\epsilon\right)$. This implies

$$
h^{T} x(t)+\delta_{1}(t)=0, \quad t \in I
$$

It is no restriction to assume that $\delta_{1}$ is monotonic on $\left[t_{1}, t_{2}\right]$. By (16)

$$
\begin{aligned}
0 & =h^{T} x(t)+\delta_{1}(t)-h^{T} x\left(t_{1}\right)-\delta_{1}\left(t_{1}\right) \\
& \geq\left|\delta_{1}(t)-\delta_{1}\left(t_{1}\right)\right|-\left|h^{T} x(t)-h^{T} x\left(t_{1}\right)\right| \\
& \geq\left(L_{\delta}-\left|h^{T}\right| \cdot L_{x}\right)\left|t-t_{1}\right|>0 .
\end{aligned}
$$


for $t \in\left(t_{1}, t_{2}\right]$, which is a contradiction. This implies that $h^{T} x(t)+\delta_{1}(t)$ moves strictly into either side of $\partial \tilde{\omega}_{1}(t)$.

\section{Gain scheduling}

Let us consider the linear system

$$
\dot{x}=L x+\gamma u
$$

with the following control law:

$$
u(x)= \begin{cases}-k_{1}^{T} x & h^{T} x>+1 \\ -k_{0}^{T} x & -1<h^{T} x<+1 \\ -k_{-1}^{T} x & h^{T} x<-1\end{cases}
$$

We can see system (17)-(18) as a hybrid system (1) with $q \in\{-1,0,1\}$ and

$$
f_{q}(x)=L_{q} x=\left(L-\gamma k_{q}^{T}\right) x
$$

and

$$
\begin{aligned}
& I_{1}^{\prime}(x)=n\left(-h^{T} x+1\right), \\
& I_{0}^{\prime}(x)=n\left(-h^{T} x-1\right) n\left(h^{T} x-1\right)=n\left(\left|h^{T} x\right|-1\right),
\end{aligned}
$$$$
I_{-1}^{\prime}(x)=n\left(h^{T} x+1\right) .
$$

The above closed loop hybrid system can be implemented by using a scalar quantizer whose input is the scalar function $h^{T} x$ and whose output is the gain of the state feedback. In quantized systems the injection of external excitation signals at the input of the quantizer is a typical solution for attenuating the distortion [11]. Thus, we consider to inject a scalar excitation signal at the quantizer input and, for the analysis of the system, it will be useful to apply the averaging theory presented so far. Actually we have the problem that, by adding the signal $\delta$ at the quantizer input $\left(h^{T} x\right)$ then we will get $\delta$ inside the absolute value of (20b) and that does not fit our model (5). It is not difficult to show that

$$
n\left(\left|h^{T} x\right|-1\right)=n\left(h^{T} x-1\right)-n\left(h^{T} x+1\right)
$$

and so doing we can reformulate our hybrid system in the following way:

$$
\begin{aligned}
I_{1}(x) & =n\left(-h^{T} x+1\right), \\
I_{0}(x) & =n\left(h^{T} x-1\right), \\
I_{-1}(x) & =n\left(h^{T} x+1\right),
\end{aligned}
$$

with

$$
\begin{aligned}
f_{1}(x) & =L_{1} x, \\
f_{0}(x) & =L_{0} x, \\
f_{-1}(x) & =\left(L_{-1}-L_{0}\right) x .
\end{aligned}
$$

Now if we add the excitation signal $\delta$ at the signal $h^{T} x$, we will get

$$
\begin{aligned}
\tilde{I}_{1}(x, \delta) & =n\left(-h^{T} x+1-\delta\right), \\
\tilde{I}_{0}(x, \delta) & =n\left(h^{T} x-1+\delta\right), \\
\tilde{I}_{-1}(x, \delta) & =n\left(h^{T} x+1+\delta\right) .
\end{aligned}
$$
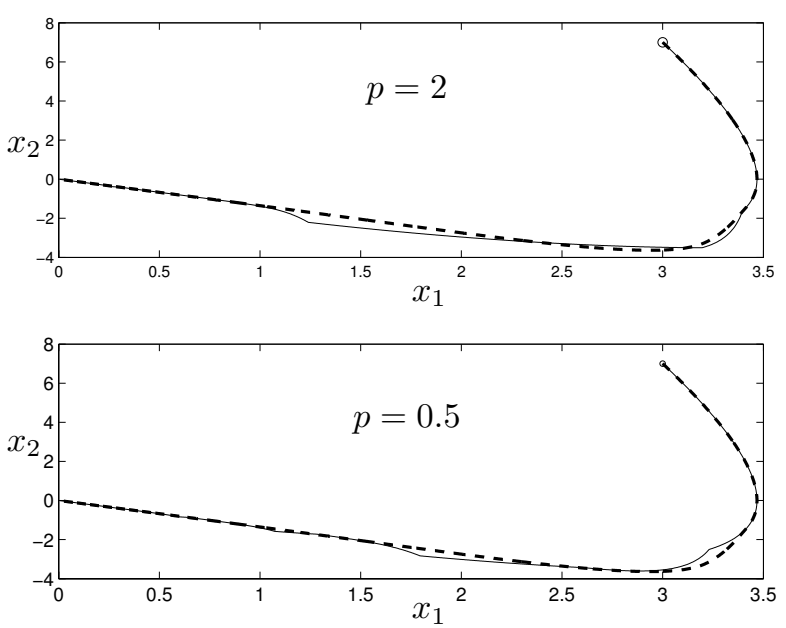

Fig. 2. State space plane of the excited hybrid system (solid) and the averaged (dotted) system with a sawtooth excitation signal with $M_{\delta}=1$ and $p=2$ (top diagram) and $p=0.5$ (bottom diagram).

Let us consider a double integrator:

$$
L=\left[\begin{array}{ll}
0 & 1 \\
0 & 0
\end{array}\right], \quad \gamma=\left[\begin{array}{l}
0 \\
1
\end{array}\right]
$$

with

$$
h^{T}=\left[\begin{array}{ll}
1 & 1
\end{array}\right] \text {. }
$$

We would like to stabilize the system by using the control law (18) with

$$
k_{1}=\left[\begin{array}{l}
7.5 \\
6.5
\end{array}\right], k_{0}=\left[\begin{array}{l}
25 \\
20
\end{array}\right], k_{-1}=\left[\begin{array}{c}
3.25 \\
3
\end{array}\right] .
$$

In this case

$$
\begin{aligned}
& L_{1}=\left[\begin{array}{cc}
0 & 1 \\
-7.5 & -6.5
\end{array}\right], L_{0}=\left[\begin{array}{cc}
0 & 1 \\
-25 & -20
\end{array}\right] \\
& L_{-1}=\left[\begin{array}{cc}
0 & 1 \\
-3.25 & -3
\end{array}\right] .
\end{aligned}
$$

We added a sawtooth excitation signal with amplitude $M_{\delta}=1$ and period $p=2$ and $p=0.5$ as explained above and Figure 2 and Figure 3 report the behaviors of the hybrid excited system and the averaged system.

\section{Sliding induced by external excitation}

Let us consider the previous example with different matrices $L_{q}$ :

$L_{1}=\left[\begin{array}{cc}0 & 1 \\ -38 & -2\end{array}\right], L_{0}=\left[\begin{array}{cc}0 & 1 \\ -26 & -2\end{array}\right], L_{-1}=\left[\begin{array}{cc}0 & 1 \\ -5 & -1\end{array}\right]$.

The chosen set of matrices does not allow sliding modes when there is no external excitation signal (see Figure 4 and Figure 5 top diagrams) but if we add a sawtooth signal $\delta$ of amplitude $M_{\delta}=1$ and period $p=0.1$ then the solution exists only over a finite time interval $t \in\left[0, t_{f}\right)$ with $t_{f} \simeq$ 1.9 (see Figure 4 and Figure 5 middle diagrams). Note that if we consider a solution in the sense of Filippov for $t>t_{f}$ we would have sliding. On the other hand, if we increase the frequency to $p=0.05$ this behavior disappears (see Figure 4 and Figure 5 bottom diagrams). 

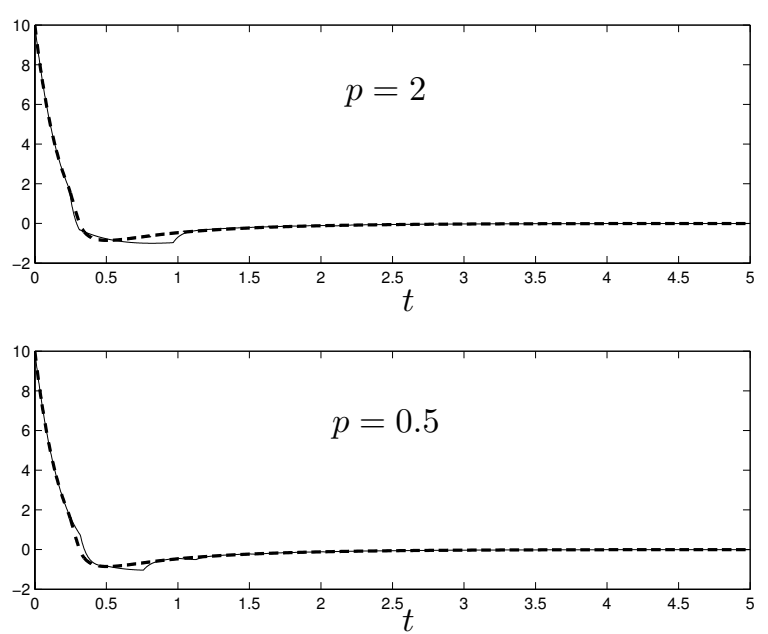

Fig. 3. Output $h^{T} x$ of the excited hybrid system (solid) and the averaged (dotted) system with a sawtooth excitation signal with $M_{\delta}=1$ and $p=2$ (top diagram) and $p=0.5$ (bottom diagram).
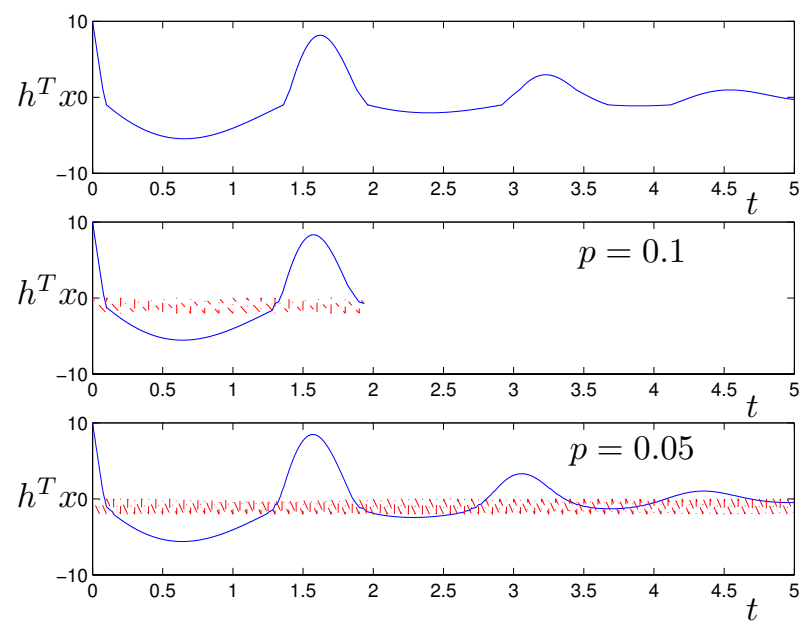

Fig. 4. Output $h^{T} x$ (solid) of the gain scheduling example without external excitation signal (top diagram), with a sawtooth excitation signal (dashed) with $p=0.1$ (middle diagram) $p=0.05$ (bottom diagram).

\section{Conclusions}

We have shown that a class of hybrid systems when forced with high frequency excitation signals can be approximated by an averaged system. The approximation is shown to be of order of the maximum repetition time interval of the excitation signal. We also indicated with an example that for sufficiently high excitation frequency, the existence of solution seems to be guaranteed even if it does not exist for low frequencies. Hybrid systems with excitation hence suggest interesting classes of systems for further well-posedness studies.The result can be exploited both for analysis and control design purposes, motivated by realistic engineering applications.

\section{REFERENCES}

[1] A. J. van der Schaft and J. M. Schumacher, "Complementarity modeling of hybrid systems," IEEE Transactions on Automatic Control, vol. 43 , no. 4 , pp. 483-490, 1998.
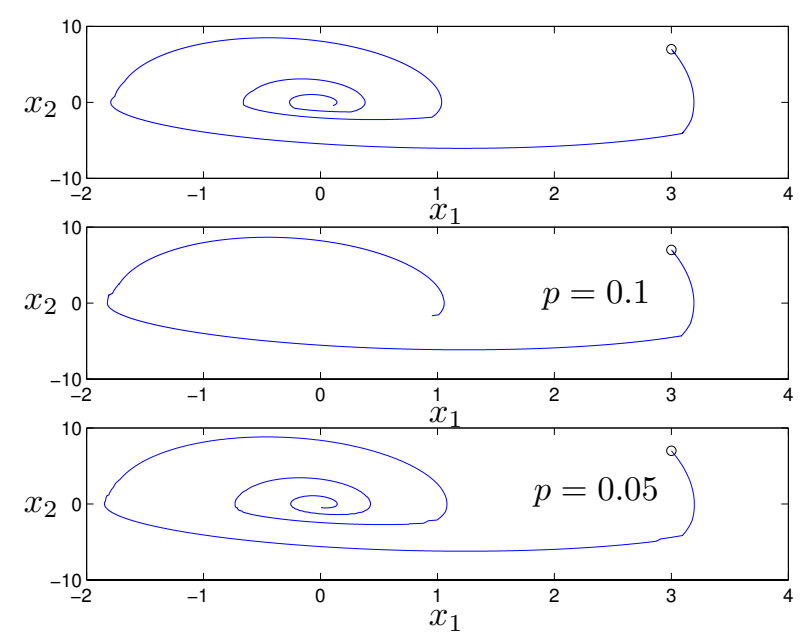

Fig. 5. State space plane of the gain scheduling example without external excitation signal (top diagram), with a sawtooth excitation signal with $p=0.1$ (middle diagram) $p=0.05$ (bottom diagram).

[2] A. Y. Pogromsky, W. P. M. H. Heemels, and H. Nijmeijer, "On solution concepts and well-posedness of linear relay systems," Automatica, vol. 39, no. 12, pp. 2139-2147, 2003.

[3] K. H. Johansson, M. Egerstedt, J. Lygeros, and S. Sastry, "On the regularization of Zeno hybrid automata," System \& Control Letters, vol. 38, pp. 141-150, 1999.

[4] M. di Bernardo, K. Johansson, and F. Vasca, "Self-oscillations and sliding in relay feedback systems: Symmetry and bifurcations," International Journal of Bifurcations and Chaos, vol. 11, no. 4, pp. 1121-1140, 2000.

[5] K. Johansson, A. Barabanov, and K. Åström, "Limit cycles with chattering in relay feedback systems," IEEE Transactions on Automatic Control, vol. 47, no. 9, pp. 1414-1423, 2002.

[6] B. Armstrong-Helouvry, P. Dupont, and C. C. de Wit, "A survey of models, analysis tools and compensation methods for control of machines with friction," Automatica, vol. 30, no. 7, pp. 1083-1138, 1994.

[7] B. Sedghi, "Control design of hybrid systems via dehybridization," Ph.D. dissertation, École Polytechnique Fédérale de Lausanne, Lausanne, Switzerland, 2003.

[8] B. Lehman and R. Bass, "Extensions of averaging theory for power electronics systems," IEEE Transactions on Power Electronics, vol. 11, no. 4, pp. 542-553, 1996.

[9] A. V. Peterchev and S. R. Sanders, "Quantization resolution and limit cycling in digitally controlled PWM converters," vol. 18, no. 1, pp. 301-308, 2003.

[10] L. Iannelli, K. Johansson, U. Jönsson, and F. Vasca, "Dither for smoothing relay feedback systems: an averaging approach," IEEE Transactions on Circuits and Systems, Part I, vol. 50, no. 8, pp. 1025-1035, 2003.

[11] H. Pan and A. Abidi, "Spectral spurs due to quantization in nyquist adcs," IEEE Transactions on Circuits and Systems - I: Regular Papers, vol. 51, no. 8, pp. 1422-1439, 2004.

[12] L. Iannelli, K. Johansson, U. Jönsson, and F. Vasca, "Dither shape in the averaging of switched systems," in Proc. of the American Control Conference, Boston, Massachussets (USA), July 2004.

[13] G. Zames and N. A. Shneydor, "Dither in non-linear systems," IEEE Transactions on Automatic Control, vol. 21, no. 5, pp. 660-667, 1976.

[14] A. Teel, L. Moreau, and D. Nesic, "Input-to-state set stability of pulse width modulated systems with disturbances," Systems and Control Letters, vol. 51, no. 1, pp. 23-32, 2004.

[15] L. Iannelli, K. Johansson, U. Jönsson, and F. Vasca, "Averaging theorem for hybrid systems with external excitation," Department of Engineering, University of Sannio, Benevento, Tech. Rep., September 2004. 